\title{
GÉNERO Y PREVENCIÓN DE DROGODEPENDENCIAS: EVALUACIÓN DEL PROGRAMA ESCOLAR "JUEGO DE LLAVES"
}

\author{
GENDER AND DRUG ABUSE PREVENTION: EVALUATION OF THE SCHOOL-BASED PROGRAM “JUEGO DE \\ LLAVES"
}

M. Aránzazu Fernández-Rodríguez, Yolanda Fontanil-Gómez, Sandra Dema-Moreno, Belén QuinteroOrdóñez e Ignacio González-López

\begin{abstract}
El consumo de drogas en menores es una realidad preocupante, especialmente el de sustancias legales. Una de las principales medidas emprendidas para hacer frente a esta situación es el desarrollo de programas de prevención escolar dirigidos a favorecer la adquisición de actitudes y estilos de vida saludable. El objetivo de este artículo es comprobar si existen diferencias en el efecto de este tipo de programas en función del género. Para ello se lleva a cabo un análisis de los datos obtenidos en la evaluación del programa escolar "Juego de Llaves" realizada en el curso escolar 2016-2017 con una muestra de 4.195 estudiantes de Educación Secundaria de 32 centros educativos. Los resultados muestran diferencias ligadas al género previas a la implantación del programa en temas como el ocio, interacción social, gestión emocional, valores e información sobre drogas, que se mantienen una vez realizado. La aplicación del programa consigue un efecto diferencial en la autoestima que se incrementa en el grupo de las adolescentes. Se concluye recomendando que cualquier programa preventivo debe analizar las desigualdades observadas y las situaciones de vulnerabilidad específicas y proponer la definición de indicadores de género que permitan medir la efectividad de los programas en ambos sexos.
\end{abstract}

Palabras clave: adolescentes, drogodependencias, evaluación de programas, género, prevención escolar.

Drug use among adolescents is a worrying reality, especially regarding legal substances. One of the main measures undertaken to face this situation is the implementation of school-based drug abuse prevention programs that are focused on promoting the acquisition of healthy attitudes and lifestyles by students. This article aims to know if there are differences in the effects of this type of programmes according to gender. For this purpose, an analysis is performed with the data obtained through the evaluation of the school-based Program "Juego de Llaves". This evaluation was carried out in the course 2016-2017 with a sample of 4.195 students of compulsory secondary education from 32 Spanish schools. The results show gender-related differences prior to the implementation of the program on topics such as leisure time, social interaction, emotional management, values and information about drugs, which are maintained once it is carried out. The application of the program achieves a differential effect on self-esteem that increases in the group of girls. It concludes by recommending that any preventive program should analyze observed inequalities and specific vulnerability situations and propose the definition of gender indicators to measure the effectiveness of programmes in both sexes.

Keywords: adolescents, substance abuse, program evaluation, gender, school-based prevention.

La adolescencia es una etapa evolutiva caracterizada por numerosos cambios físicos, psicológicos y de comportamiento, en la que se suele iniciar el desarrollo de diferentes conductas

María Aránzazu Fernández Rodríguez, Fundación C.E.S.P.A.-Proyecto Hombre Asturias, España, https://orcid.org/0000-0003-4407$\underline{8603 .}$.

Yolanda Fontanil Gómez, Universidad de Oviedo, España, https://orcid.org/0000-0002-4493-3115.

Sandra Dema Moreno, Universidad de Oviedo, España, correspondencia a demasandra@uniovi.es, https://orcid.org/0000-00031845-5962.

Belén Quintero Ordóñez, Universidad de Córdoba, España, https://orcid.org/0000-0001-7458-6591. Ignacio González López, Universidad de Córdoba, España, https://orcid.org/0000-0002-9114-4370. 
de riesgo, como el consumo de sustancias psicoactivas. La Encuesta sobre Uso de Drogas en Enseñanzas Secundarias en España (ESTUDES) realizada en el curso escolar 2018-19 señala que el $75.9 \%$ de estudiantes entre 14 y 18 años ha consumido bebidas alcohólicas en el último año, el $35 \%$ tabaco, el $27.5 \%$ cannabis y el $12.5 \%$ hipnosedantes (comúnmente conocidos como tranquilizantes o ansiolíticos) con y sin receta. Según esta encuesta, la edad media de inicio en el consumo de este tipo de sustancias se sitúa en los 14 años por término medio, siendo las chicas quienes obtienen unos mayores porcentajes, a excepción del cannabis.

Atendiendo a esta realidad, no es de extrañar que el consumo de drogas en menores sea preocupante, especialmente en lo que al uso de sustancias legales se refiere. Para hacer frente a esta situación desde algunas instituciones, principalmente del ámbito de la salud, se ha planteado el desarrollo de actuaciones de prevención de drogodependencias dirigidas a diferentes contextos (escolar, comunitario, familiar, etc.) como una de las principales medidas a emprender. A lo largo de las últimas décadas, el número y tipo de programas preventivos realizados ha aumentado de manera exponencial, siendo la prevención escolar donde se ha producido este desarrollo con una mayor intensidad debido a las características propias del contexto educativo (Gómez, Luengo \& Romero, 2002).

La escuela es el espacio idóneo en el que llevar a cabo este tipo de intervenciones, ya que se trata de uno de los principales agentes de socialización, junto a la familia y el grupo de iguales, con una clara función educativa, lo que posibilita la detección precoz de posibles factores de riesgo relacionados con el consumo de drogas (Alonso, Freijo \& Freijo, 1996). Además, permite alcanzar a toda la población en edad escolar y facilita la implementación de actuaciones dentro del horario escolar (Soole, Mazerolle, \& Rombouts, 2008).

Inicialmente, las intervenciones de prevención escolar no contemplaban la evaluación de su eficacia, pero a partir de la década de los 90 del siglo XX se han realizado numerosos estudios dirigidos a evaluar los resultados de este tipo de programas, posteriormente resumidos en varias revisiones y metanálisis, que permiten tener un conocimiento bastante exhaustivo acerca del estado de la cuestión en este ámbito. Asimismo, el establecimiento de criterios o principios de efectividad que deben incorporar este tipo de programas ha sido una práctica habitual de los organismos e instituciones públicas encargadas de las políticas sobre drogas a lo largo de las dos últimas décadas (Gandhi, Murphy-Graham, Petrosino, Chrismer \& Weiss, 2007). Por lo tanto, no es de extrañar que la evidencia más sólida en el ámbito de la prevención de drogodependencias se encuentre en las intervenciones escolares universales, esto es, las dirigidas al conjunto de la población escolar (MacArthur et al., 2018).

Son muchos los estudios que han demostrado que la prevención escolar es una estrategia eficaz para prevenir conductas de riesgo en menores como el consumo de sustancias psicoactivas, especialmente alcohol, tabaco y cannabis, y que además tiene un impacto positivo sobre determinadas variables mediadoras, asociadas con el uso de drogas, como la percepción normativa, entre otras (Gázquez, García, \& Espada, 2009; Hansen \& McNeal Jr., 1996; Isorna, 2013; Gómez et al., 2002; MacArthur et al., 2018; MacKinnon et al., 1991; MacKinnon, 1994).

Por otro lado, las revisiones críticas y metanálisis realizados sobre esta cuestión han permitido identificar componentes específicos relacionados con el éxito de los programas de prevención escolar, como son el tipo de contenidos, la intensidad del programa y la metodología de aplicación, entre otros. Las principales conclusiones recogidas en estos trabajos señalan que los programas de prevención escolar más eficaces parten de teorías psicosociales; promueven, a 
través de una metodología interactiva, el desarrollo de habilidades y competencias sociales a nivel individual; tratan de abordar los factores de riesgo y protección relacionados con el consumo de drogas; y se dirigen a las primeras etapas en las que se inicia esta conducta (Espada, Gonzálvez, Orgilés, Lloret \& Guillén-Riquelme, 2015; Faggiano et al., 2008; Gázquez et al., 2009; Hawks, Scott, McBride, Jones \& Stockwell, 2002; Onrust, Otten, Lammers \& Smit, 2016; Soole et al., 2008; Tobler et al., 2000; Wagner, Tubman \& Gil, 2004)

\section{Género y prevención de drogodependencias en el ámbito escolar}

En este proceso de mejora de la efectividad de los programas preventivos, el género no ha sido entendido como un factor claro de influencia en la investigación desarrollada. Es a finales del siglo XX, cuando diferentes instituciones de reconocido prestigio a nivel internacional en el ámbito de la salud pública, y más en concreto de las adicciones, como el National Institute on Drug Abuse (NIDA) o el Centre for Substance Abuse Prevention (CSPA), impulsan de manera considerable la investigación y el diseño de programas de tratamiento sensibles al género. Sin embargo, la prevención de drogodependencias continuó desarrollándose durante esos años sin prestar atención a esta cuestión, bajo la idea de que los programas preventivos son igual de efectivos para chicos y chicas (Blake, Amaro, Schwartz \& Flinchbaugh, 2001).

Posteriormente, la realización de investigaciones, de corte transversal y longitudinal, dirigidas a analizar el consumo de drogas en la adolescencia, permitió identificar factores de riesgo y protección específicos en función del género. Lo que evidenció, por un lado, la potencialidad del género como variable explicativa del inicio y mantenimiento en el consumo de sustancias y sus consecuencias (Guthrie et al., 2001), y por otro, la necesaria revisión de los modelos teóricos tradicionales que orientan el desarrollo de los programas preventivos para ver de qué manera se puede mejorar la efectividad de las intervenciones dirigidas a las mujeres (Amaro, Blake, Schwartz \& Flinchbaugh, 2001).

Estas cuestiones, unidas al aumento del consumo de drogas en chicas jóvenes, especialmente tabaco, alcohol y psicofármacos, llevaron a plantear la reflexión acerca de si los programas de prevención existentes son igual de efectivos en ambos sexos (Blake et al., 2001; Kumpfer, Smith \& Summerhays, 2008). Durante las dos últimas décadas se han llevado a cabo varias revisiones sistemáticas con el objetivo de conocer la efectividad de los programas de prevención tradicionales en chicas adolescentes con resultados muy variados. Algunos de ellos han demostrado ser efectivos en chicas, pero no en chicos y, a la inversa, otros parecen tener un efecto positivo en los chicos, pero negativo en las chicas (Blake et al., 2001; Kumpfer \& Magalhães, 2016; Kumpfer et al., 2008; Vigna-Taglianti et al., 2009).

La escasez de estudios en este ámbito y la diversidad de sus resultados exigen continuar desarrollando investigaciones que analicen los componentes de estos programas, con el objetivo de identificar los aspectos clave de las intervenciones que contribuyan a lograr una mayor efectividad en ambos sexos (Kumpfer et al., 2008; Kumpfer \& Magalhães, 2016). Diferentes instituciones internacionales, como la Red Canadiense de Salud de las Mujeres (CWHN), o más recientemente la Oficina de Naciones Unidas contra la Droga y el Delito (UNDOC), proponen, entre las posibles medidas para incorporar el enfoque de género en la investigación, la revisión de estudios originales con análisis secundarios complementarios que, sin modificar el diseño de la investigación, permitan determinar el impacto de los programas desde la perspectiva de género (Johnson, Greaves y Repta, 2007; Kumpfer \&Magalhães, 2016). Desde esta óptica, en el presente artículo se plantea la revisión de la evaluación de un programa de prevención escolar universal 
dirigido a alumnado de Educación Secundaria Obligatoria denominado "Juego de Llaves", con el objetivo de analizar si la variable género afecta a los resultados del mismo.

El programa "Juego de Llaves", elaborado por la Asociación Proyecto Hombre (2014), es fruto de la revisión y actualización del programa "Entre Todos" creado por esta misma entidad en el año 2000. Su diseño está fuertemente influenciado por el programa "Construyendo Salud", que es la adaptación española del anglosajón Life Skills Training, realizada por Luengo y colaboradores (1998). Life Skills Training es una de las intervenciones en el ámbito de prevención escolar con mayor historial de implementación y evaluación a lo largo de los últimos 30 años. Dicho programa, basado en el modelo de influencia social, plantea el desarrollo de la competencia individual y social como aspecto clave para prevenir el consumo de drogas en adolescentes, mediante el entrenamiento en habilidades para mejorar la autogestión (autoestima, resolución de conflictos, toma de decisiones, gestión emocional, autocontrol, etc.), la interacción social (habilidades sociales, de comunicación, asertividad, etc.) y la adopción de actitudes saludables (Botvin \& Griffin, 2014).

Bajo este marco teórico, el programa "Juego de Llaves" se compone de seis módulos que el profesorado desarrolla en el aula a través de varias sesiones, mediante una metodología interactiva. En cada uno de los módulos se incluyen contenidos señalados por la literatura existente, como factores de riesgo y protección en relación con el consumo de drogas, como los anteriormente mencionados.

Tanto el programa "Entre Todos", como su versión renovada y actualizada "Juego de Llaves", han sido evaluados. Las principales conclusiones obtenidas en la evaluación del programa original señalan su eficacia en la consecución de los objetivos relacionados con la prevención del consumo de tabaco y alcohol, así como la obtención de resultados positivos para variables intermedias o mediadoras de esta conducta, como la conducta prosocial, las actitudes e información sobre alcohol y tabaco, la asertividad, y los valores personales, entre otras (Gil, 2002). Respecto a la evaluación del programa "Juego de Llaves" los resultados sugieren un efecto positivo sobre variables como la asertividad, la identificación emocional, la información sobre drogas y la toma de decisiones razonada (Condes et al., 2018).

Al igual que ocurre con la mayor parte de las evaluaciones realizadas sobre otros programas de prevención escolar, el género no ha sido una variable fundamental en el desarrollo de este estudio. De hecho, la única aproximación diferencial a los resultados que se realiza en la evaluación del programa se relaciona con la satisfacción entre los y las adolescentes, pero no con el impacto del mismo en unos y otras. Así, las chicas son quienes mejor valoran el programa en su conjunto, y de manera específica, la labor motivacional del profesorado y los recursos de apoyo utilizados para su implantación (Condes et al., 2018).

En este artículo se realiza un análisis de los datos secundarios obtenidos a través de esta evaluación, con el objetivo de comprobar si existen diferencias entre chicos y chicas en relación con los resultados del programa "Juego de Llaves", y conocer cómo afecta la variable género a su efectividad. 
M. Aránzazu Fernández-Rodríguez, Yolanda Fontanil-Gómez, Sandra Dema-Moreno, Belén Quintero-Ordóñez e Ignacio GonzálezLópez

\section{Método}

\section{Participantes}

La población que participó en la evaluación estuvo conformada por todo el alumnado de Educación Secundaria Obligatoria que por primera vez participaba en el programa "Juego de Llaves" en el curso escolar 2016-2017, en total 4.195 estudiantes. Este alumnado procede de 32 centros educativos distribuidos en 19 localidades pertenecientes a 5 comunidades autónomas (Andalucía, Asturias, Canarias, Castilla-La Mancha y Cataluña) y a la ciudad autónoma de Melilla. Los centros eran en su mayoría de carácter público (90.3\%), siendo muy escasa la participación de alumnado de centros concertados (9.7\%). La distribución de la población en función del sexo es equilibrada, siendo la presencia de varones de un $49.9 \%$ y la de mujeres de un $50.1 \%$. En referencia al curso, y por tanto a las edades del alumnado, más de dos tercios de las personas participantes se encontraban cursando el primer ciclo de Educación Secundaria (45.9 \% de 1ㅇde ESO y $22.9 \%$ de 20 de ESO), y el tercio restante el segundo ciclo de Educación Secundaria (15.4\% de 3 o de ESO y 15.8 de 4을 de ESO). Esta diferencia de porcentajes está relacionada con el hecho de que el criterio de selección del alumnado fue la participación por primera vez en el programa. Habitualmente, los centros educativos que implementan el programa comienzan a hacerlo en el primer ciclo de Educación Secundaria y, sobre todo, en el primer curso.

\section{Instrumentos}

Para evaluar el efecto diferencial del programa "Juego de Llaves" se utilizó el cuestionario sobre las dimensiones del mismo elaborado ad hoc para llevar a cabo la evaluación original. Este instrumento está formado por 32 ítems, ocho de los cuales recogen información sobre variables sociodemográficas, y los 24 restantes evalúan las variables definitorias de cada una de las seis dimensiones de análisis establecidas ("ocio y tiempo libre", "interacción social", "gestión emocional", "estrategias cognitivas", "valores" e "información sobre drogas"). Estas dimensiones se corresponden con los seis módulos que trabaja el profesorado con el alumnado a través de las distintas sesiones desarrolladas a lo largo de un curso escolar. Tales ítems recogen el grado de acuerdo con la afirmación que contienen en una escala tipo Likert de 1 a 5, donde 1 representa el menor acuerdo y 5 el mayor. El instrumento tiene un Alfa de Cronbach de $\alpha=.652$ para el cuestionario total, y para cada uno de los ítems por encima de $\alpha=.621$. La validez se estimó por medio de una prueba de discriminación de ítems, siendo válidos el $75 \%$ y sometidos a modificación el 25\% restante (Condes et al., 2018).

\section{Procedimiento}

La participación en la evaluación se ofreció a todos los centros educativos que estaban desarrollando el programa "Juego de Llaves" durante el curso escolar 2016-2017 en el territorio nacional. Los centros interesados suscribieron voluntariamente un compromiso de participación en la investigación, en el que se especificaban las tareas asumidas, así como los datos del centro (persona responsable y número de aulas, profesorado y alumnado implicado en la evaluación).

El criterio de selección del alumnado fue su condición de novel respecto a la participación en el programa, independientemente del curso escolar que estuviera cursando. Al tratarse de menores de edad se solicitó el consentimiento informado por escrito del padre, madre o responsable legal, en el que se informaba de los objetivos de la evaluación, así como del procedimiento para garantizar la confidencialidad, protección y tratamiento de los datos. 
En cada una de las aulas que finalmente participaron en el estudio se pasó el cuestionario de forma colectiva, siendo autocumplimentado de manera anónima por el alumnado, en presencia del tutor o tutora de cada clase. El tiempo de cumplimentación aproximado fue de 15 a 20 minutos y se realizó en horario escolar.

El diseño empleado fue el de investigación evaluativa cuasiexperimental, sin grupo control, con dos mediciones: pretest y postest. La primera medición tuvo lugar en el mes de noviembre de 2016, antes de aplicar el programa. La segunda se realizó a final de ese curso escolar, durante el mes de mayo de 2017.

\section{Análisis de datos}

En este artículo se ha trabajado con los datos obtenidos en la citada evaluación, utilizando para su tratamiento el programa de análisis estadístico SPSS v.22.0. En primer lugar, se llevó a cabo un análisis de la normalidad asimetría y curtosis para posteriormente realizar un Análisis Multivariado de Varianza (MANOVA) (n.s.=.05) al objeto de conocer si existen diferencias entre chicos y chicas respecto a las dimensiones de análisis tras la aplicación del programa. Para identificar los elementos diferenciadores se llevó a cabo la aplicación de una prueba $t$ de Student para muestras independientes (n.s.=.05) entre las medias obtenidas en ambos sexos en el postest. Para medir la magnitud del efecto de las diferencias se utilizó el índice $d$ de Cohen.

\section{Resultados}

Previo a dotar de valor a los resultados obtenidos, se determinó la existencia de normalidad univariada en las medidas observadas, mediante los índices de asimetría y curtosis, salvo para los ítems emoc2 (emoc2=soy capaz de darme cuenta cuando siento miedo, tristeza, rabia y alegría) e info3 (info3=conozco las consecuencias del consumo de drogas) (Tabla 1). Asimismo, la bondad de ajuste resultó ser significativa en todos los elementos propuestos para cada una de las medidas $(p=.000)$.

Tabla 1

Índices de asimetría y curtosis en el Postest

\begin{tabular}{lcccc}
\hline & \multicolumn{2}{c}{ Postest } \\
& Asimetría & \multicolumn{2}{c}{ Curtosis } \\
\cline { 2 - 5 } & Coeficiente & $\begin{array}{c}\text { Error } \\
\text { típico }\end{array}$ & Coeficiente & Error \\
& & .040 & 3.604 & .081 \\
emoc2 & -1.974 & .041 & 2.810 & .082 \\
\hline
\end{tabular}

Para analizar la influencia de la variable género en las dimensiones de análisis se llevó a cabo un Análisis Multivariado de Varianza (MANOVA). Los resultados obtenidos ( $L$ de Wilks=.960, $F=6.233, p=.000$ ) reflejan la existencia de elementos diferenciales en atención a esta variable tras la implantación del programa.

Los resultados con la $t$ de Student para muestras independientes (n.s.>.05) revelan diferencias estadísticamente significativas entre hombres y mujeres en todas las dimensiones de análisis, a excepción de "estrategias cognitivas", y en un total de 10 ítems que aparecen en la 
M. Aránzazu Fernández-Rodríguez, Yolanda Fontanil-Gómez, Sandra Dema-Moreno, Belén Quintero-Ordóñez e Ignacio GonzálezLópez

Tabla 2. Los chicos obtienen puntuaciones medias superiores en los ítems ocio1, ocio2, ocio5, inter4 y emoc3, y las chicas en los ítems inter5, inter6, emoc1, valor3 e info4.

Tabla 2

Resultados por sexo estadísticamente significativos según $t$ de Student en el postest

\begin{tabular}{|c|c|c|c|c|c|c|c|}
\hline \multirow{3}{*}{ Ítems } & \multicolumn{7}{|c|}{ Postest } \\
\hline & \multicolumn{2}{|c|}{ Hombres } & \multicolumn{2}{|c|}{ Mujeres } & \multicolumn{3}{|c|}{ Prueba de t } \\
\hline & Media & DT & Media & DT & $\begin{array}{c}\text { Diferencia de } \\
\text { medias }\end{array}$ & $\mathrm{t}$ & $p$ \\
\hline ocio1 & 3.95 & 1.128 & 3.77 & 1.156 & .182 & 4.799 & .000 \\
\hline ocio2 & 3.68 & 1.261 & 3.57 & 1.282 & .108 & 2.556 & .011 \\
\hline ocio5 & 1.76 & 1.134 & 1.57 & 1.044 & .186 & 5.109 & .000 \\
\hline inter4 & 2.85 & 1.325 & 2.72 & 1.273 & .130 & 2.987 & .003 \\
\hline inter5 & 2.54 & 1.426 & 2.66 & 1.342 & -.125 & -2.693 & .007 \\
\hline inter6 & 3.89 & 1.139 & 4.05 & 1.046 & -.159 & -4.345 & .000 \\
\hline emoc1 & 3.89 & 1.166 & 3.99 & 1.145 & -.102 & -2.671 & .008 \\
\hline emoc3 & 3.85 & 1.163 & 3.69 & 1.153 & .157 & 4.069 & .000 \\
\hline valor3 & 3.72 & 1.075 & 3.81 & 1.041 & -.090 & -2.523 & .012 \\
\hline info4 & 4.05 & 1.308 & 4.15 & 1.214 & -.093 & -2.220 & .026 \\
\hline
\end{tabular}

Nota: ocio1=Cuando quiero buscar actividades de ocio sé a dónde dirigirme; ocio2=Conozco la oferta de actividades de ocio de mi entorno (barrio, ciudad, pueblo, etc.); ocio5=Mis amigos y/o amigas deciden las actividades de ocio en las que participo; inter4=Cuando contemplo una situación de violencia me desentiendo; inter5=Ante una situación de violencia no sé cómo actuar; inter6=Ante una situación de violencia ayudo a la persona agredida; emoc1=Creo que soy una persona valiosa; emoc3=Sé lo que hacer para manejar y controlar mis emociones; valor3=Mis actuaciones y comportamientos van en consonancia con los valores que son importantes para mí; info4=El alcohol es una droga; $p<.05$

En relación con el tamaño del efecto, señalar que todos los ítems que presentan resultados estadísticamente significativos obtienen una $d \leq .2$, que según Cohen (1988) indica un efecto pequeño, empezando a ser aceptable a partir de ese dato. Sin embargo, otros autores señalaron que el efecto de una intervención depende de sus costos y beneficios relativos, pudiendo considerarse significativo partir de $d \geq .1$ si implica una mejora sustantiva en un amplio grupo de población destinataria (Glass, McGaw y Smith, 1981). Bajo esta circunstancia, se encuentran la mitad de los ítems. Dos de ellos pertenecen a la dimensión de análisis "ocio y tiempo libre", ocio1=Cuando quiero buscar actividades de ocio sé a dónde dirigirme $(d=.158)$ y ocio5=Mis amigos y/o amigas deciden las actividades de ocio en las que participo $(d=.174)$, otros dos a "interacción social", inter4=Cuando contemplo una situación de violencia me desentiendo $(d=.1)$ e inter6=Ante una situación de violencia ayudo a la persona agredida $(d=.125)$, y uno a "gestión emocional", emoc3=Sé lo que hacer para manejar y controlar mis emociones ( $d=.138)$.

Con el objetivo de conocer si esta situación diferencial entre chicos y chicas puede atribuirse al programa se estableció una comparación con las diferencias estadísticamente significativas encontradas para ambos sexos en el pretest. Se observó que en todos los ítems en los que se encontraron diferencias en el postest, también se identificaron diferencias en el pretest, a excepción de emoc1=Creo que soy una persona valiosa, donde solo aparecen tras la aplicación del programa (Tabla 3). 
Tabla 3

Comparación de resultados estadísticamente significativos según t de Student en el pretest y/o postest por sexo

\begin{tabular}{|c|c|c|c|c|c|c|c|c|}
\hline \multirow{3}{*}{ Ítems } & \multicolumn{4}{|c|}{ Pretest } & \multicolumn{4}{|c|}{ Postest } \\
\hline & \multirow{2}{*}{$\begin{array}{c}\text { Hombres } \\
\mathrm{M}(\mathrm{DT})\end{array}$} & \multirow{2}{*}{$\begin{array}{l}\text { Mujeres } \\
M(D T)\end{array}$} & \multicolumn{2}{|c|}{ Prueba de $t$} & \multirow{2}{*}{$\begin{array}{c}\text { Hombres } \\
\text { M (DT) }\end{array}$} & \multirow{2}{*}{$\begin{array}{l}\text { Mujeres } \\
\text { M (DT) }\end{array}$} & \multicolumn{2}{|c|}{ Prueba de $t$} \\
\hline & & & Df & $t(p)$ & & & Df & $\mathrm{t}(p)$ \\
\hline ocio1 & $\begin{array}{c}3.99 \\
(1.094)\end{array}$ & $\begin{array}{c}3.82 \\
(1.123)\end{array}$ & .176 & $\begin{array}{l}5.064 \\
(.000)\end{array}$ & $\begin{array}{c}3.95 \\
(1.128)\end{array}$ & $\begin{array}{c}3.77 \\
(1.156)\end{array}$ & .182 & $\begin{array}{l}4.799 \\
(.000)\end{array}$ \\
\hline ocio 2 & $\begin{array}{c}3.65 \\
(1.293)\end{array}$ & $\begin{array}{c}3.56 \\
(1.264)\end{array}$ & .090 & $\begin{array}{l}2.244 \\
(.025)\end{array}$ & $\begin{array}{c}3.68 \\
(1.261)\end{array}$ & $\begin{array}{c}3.57 \\
(1.282)\end{array}$ & .108 & $\begin{array}{l}2.556 \\
(.011)\end{array}$ \\
\hline ocio5 & $\begin{array}{c}1.75 \\
(1.167)\end{array}$ & $\begin{array}{c}1.57 \\
(1.026)\end{array}$ & . 182 & $\begin{array}{l}5.285 \\
(.000)\end{array}$ & $\begin{array}{c}1.76 \\
(1.134)\end{array}$ & $\begin{array}{c}1.57 \\
(1.044)\end{array}$ & .186 & $\begin{array}{l}5.109 \\
(.000)\end{array}$ \\
\hline inter4 & $\begin{array}{c}2.84 \\
(1.360)\end{array}$ & $\begin{array}{c}2.73 \\
(1.379)\end{array}$ & .112 & $\begin{array}{l}2.584 \\
(.010)\end{array}$ & $\begin{array}{c}2.85 \\
(1.325)\end{array}$ & $\begin{array}{c}2.72 \\
(1.273)\end{array}$ & .130 & $\begin{array}{l}2.987 \\
(.003)\end{array}$ \\
\hline inter5 & $\begin{array}{c}2.53 \\
(1.435)\end{array}$ & $\begin{array}{c}2.68 \\
(1.343)\end{array}$ & -.145 & $\begin{array}{l}-3.316 \\
(.001)\end{array}$ & $\begin{array}{c}2.54 \\
(1.426)\end{array}$ & $\begin{array}{c}2.66 \\
(1.342)\end{array}$ & -.125 & $\begin{array}{r}-2.693 \\
(.007)\end{array}$ \\
\hline inter6 & $\begin{array}{c}3.97 \\
(1.164)\end{array}$ & $\begin{array}{c}4.07 \\
(1.104)\end{array}$ & -.097 & $\begin{array}{l}-2.723 \\
(.007)\end{array}$ & $\begin{array}{c}3.89 \\
(1.139)\end{array}$ & $\begin{array}{c}4.05 \\
(1.046)\end{array}$ & -.159 & $\begin{array}{r}-4.345 \\
(.000)\end{array}$ \\
\hline emoc1 & $\begin{array}{c}3.90 \\
(1.148)\end{array}$ & $\begin{array}{c}3.94 \\
(1.145)\end{array}$ & -.034 & $\begin{array}{l}-0.947 \\
(.344)\end{array}$ & $\begin{array}{c}3.89 \\
(1.166)\end{array}$ & $\begin{array}{c}3.99 \\
(1.145)\end{array}$ & -.102 & $\begin{array}{r}-2.671 \\
(.008)\end{array}$ \\
\hline emoc3 & $\begin{array}{c}3.92 \\
(1.117)\end{array}$ & $\begin{array}{c}3.76 \\
(1.150)\end{array}$ & . 167 & $\begin{array}{l}4.714 \\
(.000)\end{array}$ & $\begin{array}{c}3.85 \\
(1.163)\end{array}$ & $\begin{array}{c}3.69 \\
(1.153)\end{array}$ & .157 & $\begin{array}{l}4.069 \\
(.000)\end{array}$ \\
\hline valor3 & $\begin{array}{c}3.78 \\
(1.050)\end{array}$ & $\begin{array}{c}3.86 \\
(1.007)\end{array}$ & -.086 & $\begin{array}{l}-2.644 \\
(.008)\end{array}$ & $\begin{array}{c}3.72 \\
(1.075)\end{array}$ & $\begin{array}{c}3.81 \\
(1.041)\end{array}$ & -.090 & $\begin{array}{r}-2.523 \\
(.012)\end{array}$ \\
\hline info4 & $\begin{array}{c}3.88 \\
(1.420)\end{array}$ & $\begin{array}{c}4.02 \\
(1.313)\end{array}$ & -.135 & $\begin{array}{l}-3.146 \\
(.002)\end{array}$ & $\begin{array}{c}4.05 \\
(1.308)\end{array}$ & $\begin{array}{c}4.15 \\
(1.214)\end{array}$ & -.093 & $\begin{array}{l}-2.220 \\
(.026)\end{array}$ \\
\hline
\end{tabular}

Nota: Df= Diferencia de medias; M=Media; ocio1=Cuando quiero buscar actividades de ocio sé a dónde dirigirme; ocio2=Conozco la oferta de actividades de ocio de mi entorno (barrio, ciudad, pueblo, etc.); ocio5=Mis amigos y/o amigas deciden las actividades de ocio en las que participo; inter4=Cuando contemplo una situación de violencia me desentiendo; inter5=Ante una situación de violencia no sé cómo actuar; inter6=Ante una situación de violencia ayudo a la persona agredida; emoc1=Creo que soy una persona valiosa; emoc3=Sé lo que hacer para manejar y controlar mis emociones; valor3=Mis actuaciones y comportamientos van en consonancia con los valores que son importantes para mí; info4=El alcohol es una droga; $p<.05$.

\section{Discusión}

A través del desarrollo de esta investigación se llevó a cabo un análisis secundario de la evaluación realizada al programa de prevención escolar "Juego de Llaves", con el objetivo de conocer si existen diferencias entre chicos y chicas, y si la variable género influye en la efectividad del mismo. En la citada evaluación se establecieron seis dimensiones de análisis ("ocio y tiempo libre", "interacción social", "gestión emocional", "estrategias cognitivas", "valores" e "información sobre drogas"), las cuales se corresponden con los seis módulos que componen el programa. En cada uno de ellos el profesorado trabaja con el alumnado contenidos señalados por 
la evidencia científica como factores de riesgo y protección relacionados con el consumo de drogas a lo largo de todo un curso escolar.

Los resultados tras la aplicación del programa muestran una situación diferencial entre ambos sexos en todas las dimensiones de análisis establecidas, a excepción de la dimensión "estrategias cognitivas". Las diferencias encontradas en la dimensión "ocio y tiempo libre" señalan que los chicos tienen un mayor conocimiento de la oferta de actividades de ocio de su entorno y de los espacios en los que buscar este tipo de información. A pesar de ello, las chicas expresan tener una mayor autonomía, respecto a su grupo de iguales, en la toma de decisiones relacionada con la elección de este tipo de actividades. En la dimensión "interacción social", los resultados muestran diferencias respecto a la reacción que tienen ambos sexos ante una situación de violencia. Los chicos en mayor medida señalan que ante este tipo de sucesos se desentienden, mientras que ellas refieren más frecuentemente no saber cómo actuar o que ayudan a la persona agredida. También aparecen diferencias en la dimensión "gestión emocional" relacionadas con un mejor manejo y control de las emociones por parte de los adolescentes, y una mayor autoestima en las chicas. En relación con la dimensión "valores", los datos señalan que la consonancia entre actuaciones y valores es más importante para ellas. La última diferencia encontrada, enmarcada en la dimensión "información sobre drogas", apunta un mayor acuerdo por parte de las adolescentes con la afirmación de que el alcohol es una droga.

Comparando estos resultados con los datos obtenidos antes de la aplicación del programa se ha podido comprobar que la única diferencia encontrada entre ambos sexos que ha aparecido una vez finalizada la intervención preventiva y que, por tanto, podría atribuirse a un efecto del programa, es la relacionada con la autoestima. En este sentido, se observa que las chicas, en mayor medida que los chicos, han mejorado la consideración que tienen de sí mismas. Este resultado es congruente con la literatura científica que señala que los programas preventivos que incorporan este componente podrían tener una mayor efectividad en las adolescentes, ya que la existencia de una baja autoestima es un factor de riesgo que afecta en mayor medida a las chicas, aumentando su vulnerabilidad respecto al uso de drogas (Amaro et al., 2001; Kumpfer \& Magalhães, 2016).

El resto de diferencias señaladas anteriormente entre los chicos y chicas adolescentes existían antes de la aplicación del programa "Juego de Llaves", por lo que el programa ha sido ciego desde el punto de vista del género, ya que no se ha conseguido modificar la situación diferencial existente entre ambos sexos respecto a varios de los componentes de la intervención preventiva y, por consiguiente, en relación con determinados factores de riesgo asociados a las drogodependencias en los que se pretende incidir a través del programa.

En esta línea, diferentes estudios afirman que los factores de riesgo y protección asociados al consumo abusivo de drogas actúan de manera diferente en chicos y chicas (López \& RodríguezArias, 2010; Schinke, Fang \& Cole, 2008). Asimismo, se ha comprobado la influencia de las nociones tradicionales de masculinidad y feminidad en el uso y gestión del ocio y tiempo libre, especialmente en la elección de las actividades y el tiempo destinado a ellas (Gradaílle, Merelas \& Garrido, 2010; Hernando, Oliva \& Pertegal, 2013); en las estrategias de interacción social y el comportamiento mostrado ante determinadas situaciones de riesgo, como la violencia entre iguales (Morales, Yubero \& Larrañaga, 2016); en la gestión de estados emocionales que pueden producir sintomatología de tipo psicológico relacionada con trastornos de ansiedad o estrés, entre otros, que se configuran como precursores del uso de drogas (Sánchez, 2014); así como en 
la percepción de riesgo asociada a las sustancias psicoactivas, y el valor que se atribuye a la salud y al autocuidado (Artazcoz \& Borrell, 2007).

Atendiendo a estos datos, y a pesar de que el programa hace mención explícita a la incorporación de la perspectiva de género en su diseño, se podría decir que no se han incorporado de manera efectiva las necesidades específicas de chicos y chicas en relación con la prevención de los consumos de drogas. Esta conclusión es congruente con otros estudios que señalan, por un lado, que la mayoría de los programas preventivos que se desarrollan, a pesar de estar diseñados siguiendo la evidencia científica, no prestan suficiente atención al género y deberían mejorar el proceso a través del cual tratan de incluir dicha perspectiva (Blake et al., 2001; European Monitoring Centre for Drugs and Drug Addiction, 2006; Fernández, Dema \& Fontanil, 2020; Kumpfer et al., 2008). Y, por otro, que la incorporación de componentes que abordan situaciones de vulnerabilidad específicas en función del género en los programas de prevención universal podría mejorar su efectividad en ambos sexos (Kumpfer \& Magalhães, 2016; Novák, Miovský, Gabrhelík, Štastná \& Jurystová, 2013; Vigna-Taglianti et al. 2009). En este sentido, recientes estudios han apuntado la necesidad de considerar nuevas vulnerabilidades que surgen en los contextos de ocio en los que se produce un consumo abusivo de drogas, fundamentalmente alcohol, y un uso continuo de dispositivos tecnológicos como el móvil, que pueden derivar en una mayor exposición de las chicas a la violencia sexual y tecnológica (RomoAvilés, García-Carpintero \& Pavón-Benítez, 2019).

Los resultados obtenidos deben ser interpretados teniendo en cuenta las limitaciones de este trabajo, relacionadas principalmente con el diseño de la investigación original. Al tratarse de una evaluación de corte transversal, no se ha podido comprobar si las diferencias encontradas tras la aplicación del programa en relación con la autoestima se mantienen en el tiempo. Tampoco se ha podido llevar a cabo un análisis intragrupal en función del sexo que permitiera observar si existían diferencias estadísticamente significativas antes y después de la intervención. Finalmente, señalar que hubiera sido interesante medir los efectos del programa sobre el uso y/o la intención de consumo de drogas, así como las diferencias entre ambos sexos a este respecto, pero desafortunadamente los datos de la evaluación original impiden realizar este análisis.

A pesar de las limitaciones, en este artículo se pone de manifiesto la necesidad de mejorar el proceso de incorporación del enfoque de género en los programas de prevención de drogodependencias, a nivel general, y de manera específica en los dirigidos al contexto escolar. Para alcanzar este objetivo es fundamental aplicar el género de forma transversal en todas las fases que componen el proceso de elaboración de una intervención preventiva. Para ello, sería recomendable la realización de una evaluación previa que permita identificar las diferencias y desigualdades entre hombres y mujeres respecto a los componentes que se van a trabajar, la incorporación de objetivos y componentes específicos dirigidos a modificar las desigualdades observadas y las situaciones de vulnerabilidad específicas en ambos sexos, así como la definición de indicadores de género que permitan medir la efectividad del programa para unas y otros.

\section{Agradecimientos}

Para la realización de este artículo se han utilizado los datos obtenidos en la evaluación del programa de prevención escolar y familiar "Juego de Llaves". Las autoras agradecen la cesión de los datos para llevar a cabo este trabajo a la Asociación Proyecto Hombre, promotora de la evaluación, y a los Centros asociados de Proyecto Hombre de Asturias, Cádiz, Canarias, Castilla- 
M. Aránzazu Fernández-Rodríguez, Yolanda Fontanil-Gómez, Sandra Dema-Moreno, Belén Quintero-Ordóñez e Ignacio GonzálezLópez

La Mancha, Cataluña y Málaga, encargados de la recogida de información en los distintos centros escolares participantes.

\section{Referencias}

Alonso, D., Freijo, E., \& Freijo, A. (1996). Actuar es posible. La prevención de las drogodependencias en la comunidad escolar. Madrid: Ministerio del Interior. Delegación del gobierno para el Plan Nacional sobre Drogas.

Amaro, H., Blake, S. M., Schwartz, P. M., \& Flinchbaugh, L. J. (2001). Developing theory-based substance abuse prevention programs for young adolescent girls. The Journal of Early Adolescence, 21(3), 256-293. doi:10.1177/0272431601021003002

Artazcoz, L., \& Borrell, C. (2007). Género y Salud. En I. Nerín, y M. Jané, (Ed.). Libro blanco sobre mujeres y tabaco: Abordaje con una perspectiva de género (pp. 9-22). Zaragoza: Comité para la Prevención del Tabaquismo y Ministerio de Sanidad y Consumo.

Asociación Proyecto Hombre (2000). Programa de prevención escolar y familiar Entre Todos. Madrid: Asociación Proyecto Hombre.

Asociación Proyecto Hombre (2014). Juego de Llaves. Actualización del programa de prevención escolar y familiar Entre Todos. Madrid: Asociación Proyecto Hombre.

Blake, S. M., Amaro, H., Schwartz, P. M., \& Flinchbaugh, L. J. (2001). A review of substance abuse prevention interventions for young adolescent girls. The Journal of Early Adolescence, 21(3), 294-324. doi:10.1177/0272431601021003003

Botvin, G. J., \& Griffin, K. W. (2014). Life skills training: preventing substance misuse by enhancing individual and social competence. New Directions for Youth Development, 2014(141), 5765. doi:10.1002/yd.20086

Cohen, J. (1988). Statistical Power Analysis for the Behavioral Sciences. Hillsdate, NJ: Lawrence Eslabaum Associates.

Condes, N., Fernández, M. A., Gonzálvez, E., Granero, Y., Lorenzo, M., Pérez, V.,... Quintero, B. (2018). Informe de evaluación del programa de prevención escolar y familiar Juego de Llaves. Recuperado de http://archivo.proyectohombre.es/wpcontent/uploads/2018/03/Informe-evaluacion-Juego-de-Llaves vf dl.pdf

Espada, J. P., Gonzálvez, M. T., Orgilés, M., Lloret, D., \& Guillén-Riquelme, A. (2015). Meta-analysis of the effectiveness of school substance abuse prevention programs in Spain. Psicothema, 27(1), 5-12. doi:10.7334/psicothema2014.106

European Monitoring Centre for Drugs and Drug Addiction. (2006). Annual Report 2016, Selected Issues 2: A gender perspective on drug use and responding to drug problems. Lisbon: European Monitoring Centre for Drugs and Drug Addiction.

Faggiano, F., Vigna-Taglianti, F.D., Versino, E., Zambon, A., Borraccino, A., \& Lemma, P. (2008). School-based prevention for illicit drugs use: A systematic review. Preventive Medicine, 46, 385-396. doi:10.1016/j.ypmed.2007.11.012 
Fernández, M. A., Dema, S., \& Fontanil, Y. (2020) Género y políticas sobre drogas en España: avances y limitaciones. Revista española de drogodependencias, 45 (1), 10-25.

Gandhi, A. G., Murphy-Graham, E., Petrosino, A., Chrismer, S. S., \& Weiss, C. H. (2007). The devil is in the details: Examining the evidence for "proven" school-based drug abuse prevention programs. Evaluation Review, 31(1), 43-74. doi:10.1177/0193841X06287188

Gázquez, M., García, J. A., \& Espada, J. P. (2009). Características de los programas eficaces para la prevención escolar del consumo de drogas. Salud y Drogas, 9(2), 185-208.

Gil, E. (2002). Evaluación del programa de prevención escolar y familiar. "Entre todos". Proyecto Hombre: Revista de la Asociación Proyecto Hombre, (43), 27-38.

Glass, G. V., McGaw, B., \& Smith, L. (1981). Meta-analysis in social research. London: Sage.

Gómez, J. A., Luengo, Á., \& Romero, E. (2002). Prevención del consumo de drogas en la escuela: cuatro años de seguimiento de un programa. Psicothema, 14(4), 685-692.

Gradaílle, R., Merelas, T., \& Garrido, U. (2010). Los tiempos libres de la infancia: hacia un ocio coeducativo. En T. Hernández (Presidencia) Treinta años de sociedad, treinta años de Sociología. Conferencia llevada a cabo en el X Congreso Español de Sociología, Navarra, España. Recuperado de http://www.fes-sociologia.com/files/congress/10/grupostrabajo/ponencias/515.pdf

Guthrie, B. J., Rotheram, M. J., Genero, N., Amaro, H., Chesney-Lind, M., \& Flinchbaugh, L. J. (2001). A guide to understanding female adolescents' substance abuse: Gender and ethnic considerations for prevention and treatment policy. Rockville, MD: US Department of Health and Human Services.

Hansen, W. B., \& McNeal Jr, R. B. (1996). The law of maximum expected potential effect: Constraints placed on program effectiveness by mediator relationships. Health Education Research, 11(4), 501-507.

Hawks, D., Scott, K., McBride, N., Jones, P., \& Stockwell, T. (2002). Prevention of psychoactive substance use: A selected review of what works in the area of prevention. Switzerland: World Health Organization.

Hernando, Á., Oliva, A., \& Pertegal, M. Á. (2013). Diferencias de género en los estilos de vida de los adolescentes. Psychosocial Intervention, 22(1), 15-23. doi:10.5093/in2013a3

Isorna, M. (2013). Contribuciones de la evidencia científica para la mejora de la prevención de las drogodependencias en el ámbito escolar. Revista Española de Drogodependencias, 38 (2), 117-138.

Johnson, J., Greaves, L., \& Repta, R. (2007). Better Science with Sex and Gender: A Primer for Health Research. Vancouver: Women's Health Research Network.

Kumpfer, K., \& Magalhães, C. (2016). Guidelines on drug prevention and treatment for girls and women. Recuperado de https://www.unodc.org/documents/drug-prevention-andtreatment/unodc_2016_drug_prevention_and_treatment_for_girls_and_women_E.pdf 
M. Aránzazu Fernández-Rodríguez, Yolanda Fontanil-Gómez, Sandra Dema-Moreno, Belén Quintero-Ordóñez e Ignacio GonzálezLópez

Kumpfer, K., Smith, P., \& Summerhays, J. F. (2008). A wakeup call to the prevention field: Are prevention programs for substance use effective for girls?. Substance Use \& Misuse, 43(89), 978-1001. doi:10.1080/10826080801914261

López, S., \& Rodríguez-Arias, J. L. (2010). Factores de riesgo y de protección en el consumo de drogas en adolescentes y diferencias según edad y sexo. Psicothema, 22(4), 568-573.

Luengo, A., Gómez, J.A., Garra, A., Romero, E., \& Otero, J.M. (1998). Construyendo Salud. Promoción del desarrollo personal y social. Madrid: Ministerio de Educación y Cultura.

MacArthur, G., Caldwell, D. M., Redmore, J., Watkins, S. H., Kipping, R., White, J.,... Campbell, R. (2018). Individual-, family-, and school-level interventions targeting multiple risk behaviours in young people. Cochrane Database of Systematic Reviews, (10). doi:10.1002/14651858.CD009927.pub2

Mackinnon, D. P. (1994). Analysis of mediating variables in prevention and intervention research. En A. Cazares, \& L. Beatty (Ed.), Scientific Methods for Prevention Intervention Research (pp. 127-153). Rockville, MD: US Department of Health and Human Services.

Mackinnon, D. P., Johnson, C. A., Pentz, M. A., Dwyer, J. H., Hansen, W. B., Flay, B. R., \& Wang, E. Y. I. (1991). Mediating mechanisms in a school-based drug prevention program: first-year effects of the Midwestern Prevention Project. Health Psychology, 10(3), 164-172. doi:10.1037/0278-6133.10.3.164

Morales, J. F., Yubero, S., \& Larrañaga, E. (2016). Gender and bullying: Application of a threefactor model of gender stereotyping. Sex Roles, 74(3-4), 169-180. doi:10.1007/s11199015-0463-3

Novák, P., Miovský, M., Gabrhelík, R., Št́astná, L., \& Jurystová, L. (2013). Gender-specific effectiveness of the unplugged prevention intervention in reducing substance use among Czech adolescents. Czech Sociological Review, 49(6), 903-926.

Observatorio Español de las Drogas y las Adicciones. (2019). ESTUDES 2018-19. Encuesta sobre Uso de Drogas en Enseñanzas Secundarias en España (ESTUDES) 1994-2018. Recuperado de

https://pnsd.sanidad.gob.es/profesionales/sistemas/nformacion/sistemalnformacion/pdf /ESTUDES 2018-19 Informe.pdf

Onrust, S. A., Otten, R., Lammers, J., \& Smit, F. (2016). School-based programmes to reduce and prevent substance use in different age groups: What works for whom? Systematic review and meta-regression analysis. Clinical Psychology Review, 44, 45-59. doi:10.1016/j.cpr.2015.11.002

Romo-Avilés, N., García-Carpintero, M. Á., \& Pavón-Benítez, L. (2019). Not without my mobile phone: alcohol binge drinking, gender violence and technology in the Spanish culture of intoxication. Drugs: Education, Prevention and Policy, 27(2), 154-164. doi:10.1080/09687637.2019.1585759 
Sánchez, L. (2014). Prevención del consumo de drogas con perspectiva de género recomendaciones con base en la evidencia. Guía para el desarrollo de programas de prevención. Alicante: Diputación de Alicante.

Schinke, S. P., Fang, L., \& Cole, K. C. (2008). Substance use among early adolescent girls: Risk and protective factors.Journal of Adolescent Health, 43(2), 191-194. doi:10.1016/j.jadohealth.2007.12.014

Soole, D. W., Mazerolle, L., \& Rombouts, S. (2008). School-based drug prevention programs: A review of what works. Australian \& New Zealand Journal of Criminology, 41(2), 259-286.

Tobler, N. S., Roona, M. R., Ochshorn, P., Marshall, D. G., Streke, A. V., \& Stackpole, K. M. (2000). School-based adolescent drug prevention programs: 1998 meta-analysis. Journal of Primary Prevention, 20(4), 275-336.

Vigna-Taglianti, F., Vadrucci, S., Faggiano, F., Burkhart, G., Siliquini, R., Galanti, M. R., \& EU-Dap Study Group. (2009). Is universal prevention against youths' substance misuse really universal? Gender-specific effects in the EU-Dap school-based prevention trial. Journal of Epidemiology \& Community Health, 63(9), 722-728. doi:10.1136/jech.2008.081513

Wagner, E. F., Tubman, J. G., \& Gil, A. G. (2004). Implementing school-based substance abuse interventions: Methodological dilemmas and recommended solutions. Addiction, 99, 106119. 